\title{
The combination of hawthorn extract and camphor significantly increases blood pressure: A meta-analysis and systematic review
}

\author{
Dezső Csupor $^{\mathrm{a}, \mathrm{b}, *}$, Réka Viczián ${ }^{\mathrm{a}}$, Tamás Lantos ${ }^{\mathrm{c}}$, Tivadar Kiss ${ }^{\mathrm{a}, \mathrm{b}}$, Péter Hegyi ${ }^{\mathrm{d}, \mathrm{e}}$, Judit Tenk ${ }^{\mathrm{d}}$, \\ László Márk Czumbel ${ }^{\mathrm{f}}$, Sang-ngoen Thanyaporn ${ }^{\mathrm{f}}$, Zoltán Gyöngyi ${ }^{\mathrm{g}}$, Gábor Varga ${ }^{\mathrm{f}}$, Gábor Gerber ${ }^{\mathrm{h}}$, \\ Erika Pétervári ${ }^{\mathrm{d}}$, Barbara Tóth ${ }^{\mathrm{a}, \mathrm{b}}$ \\ a Department of Pharmacognosy, Faculty of Pharmacy, University of Szeged, Szeged 6720, Hungary \\ ${ }^{\mathrm{b}}$ Interdisciplinary Centre of Natural Products, University of Szeged, Szeged 6720, Hungary \\ ${ }^{\mathrm{c}}$ Department of Medical Physics and Informatics, Faculty of Medicine, University of Szeged, Szeged 6720, Hungary \\ ${ }^{\mathrm{d}}$ Institute for Translational Medicine, Medical School, Szentágothai Research Centre, University of Pécs, Pécs 7624, Hungary \\ ${ }^{\mathrm{e}}$ MTA-SZTE Translational Gastroenterology Research Group, First Department of Medicine, University of Szeged, Szeged, Hungary \\ ${ }^{\mathrm{f}}$ Department of Oral Biology, Semmelweis University, Budapest 1089, Hungary \\ ${ }^{\mathrm{g}}$ Department of Public Health Medicine, Medical School, University of Pécs, Pécs 7624, Hungary \\ ${ }^{\mathrm{h}}$ Department of Anatomy, Histology and Embryology, Semmelweis University, Budapest 1085, Hungary
}

\section{A R T I C L E I N F O}

\section{Keywords:}

Hawthorn

Crataegus

Camphor

Hypotension

Cognitive

Meta-analysis

\begin{abstract}
A B S T R A C T
Background: A fixed combination of hawthorn and camphor (Korodin Herz-Kreislauf-Tropfen ${ }^{\circ}$ ) has been used in the therapy of hypotension for decades. Although its efficacy was evaluated in clinical trials, these studies have not been critically assessed in meta-analyses.

Purpose: To systematically evaluate the efficacy of a fix combination of camphor and hawthorn extract (Korodin ${ }^{\circ}$ ) on blood pressure and cognition compared to placebo, in a meta-analysis based on randomized controlled trials (RCTs).

Study design: The meta-analysis was carried out following the PRISMA guidelines, using the PICO format, and it was registered in the PROSPERO register.

Methods: The Cochrane Central Register of Controlled Trials, PubMed, Embase, and Web of Science databases were searched for relevant studies. Placebo-controlled clinical studies involving adult patients receiving a fix combination of hawthorn extract and camphor were included. No language or publication year restrictions were applied.

Results: Four randomized trials including a total of 221 patients were pooled for statistical analysis. According to the present meta-analysis, the fixed combination of hawthorn and camphor significantly increases systolic and diastolic blood pressure compared to placebo ( $p$-values: 0.017 and 0.049 , respectively) and had a beneficial, but not statistically significant effect on the cognitive performance in the connect-the-numbers test ( $p$-value: 0.071$)$. Conclusion: Korodin ${ }^{\infty}$ is an effective and presumably safe complementary therapy for the treatment of hypotension. Its blood pressure increasing effect is confirmed; however, the evidence supporting its use is very limited. The optimum dose and duration of treatment is still unclear. The comprehensive evaluation of efficacy and safety is required in further, high-quality clinical studies, involving larger patient populations and comparable endpoints.
\end{abstract}

\section{Introduction}

By definition, hypotension is abnormally low blood pressure, with a generally acknowledged upper limit of $90 / 60 \mathrm{mmHg}$. Orthostatic hypotension is defined as a drop of at least $20 \mathrm{mmHg}$ in systolic and/or $10 \mathrm{~mm} \mathrm{Hg}$ in diastolic blood pressure (BP) within $3 \mathrm{~min}$ of standing or

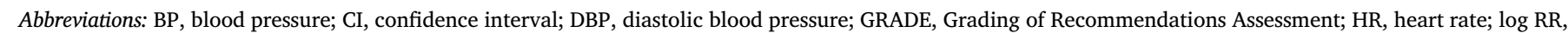

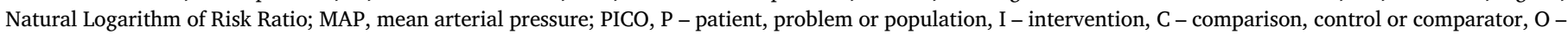
outcome; PRISMA, Preferred Reporting Items for Systematic Reviews and Meta-Analyses; RCT, randomized controlled clinical trial; SBP, systolic blood pressure

* Corresponding author.

E-mail address: csupor.dezso@pharmacognosy.hu (D. Csupor). 
head-up tilt to at least $60^{\circ}$ on a tilt table (Freeman et al., 2011). Exsiccosis, serious medical or surgical disorders, as well as taking certain medications can result in hypotension. Signs and symptoms of hypotension include dizziness, headache, nausea, palpitations, and fainting. Beyond a decreased quality of life, chronic hypotension might decrease cognitive performance as well. Serious hypotension is associated with a high risk of morbidity and mortality, and in severe cases it can be lifethreatening.

The course of treatment depends on the exact type of hypotension and on the underlying conditions. In some cases, life-style changes are introduced to normalize blood pressure, and to avoid the sudden drop of blood pressure. For the treatment of orthostatic hypotension, the first-line drug is midodrine, an alpha-adrenergic receptor agonist, which is a frequently used vasopressor. In patients with orthostatic hypotension, it elevates standing, sitting, and supine systolic and diastolic blood pressure. Droxidopa, a synthetic precursor of norepinephrine, is approved for the treatment of symptomatic neurogenic orthostatic hypotension. Although several other pharmacological interventions are also available, the quality of evidence on their counterbalancing blood pressure drop is weak (Eschlböck et al., 2017).

Apart from synthetic drugs, plants and natural products with different mechanisms of actions are also used in the treatment of hypotension. However, in most cases the effectiveness of these products is not sufficiently established. In a small, single-blind, placebo-controlled study, moderate doses $(5.4 \mathrm{mg})$ of yohimbine were found to increase systolic blood pressure of patients suffering from orthostatic hypotension (Jordan et al., 1998). Dihydroergotamine also exerts an alphaadrenergic receptor agonist activity, and based on human studies it increases arterial blood pressure in orthostatic hypotension (Victor and Talman, 2002).

A fixed combination of Crataegus (hawthorn) extract and camphor (Korodin Herz-Kreislauf-Tropfen ${ }^{\circ}$ ) has been used in patients with orthostatic hypotension and chronic hypotension for decades. In Germany Korodin is marketed as a medicine, and it contains a fluid extract of fresh Crataegus berries (DER: 1:1.3-1.5; extraction solvent: ethanol $93 \mathrm{~V} / \mathrm{V} \%, 97.3 \mathrm{~g} / 100 \mathrm{~g})$ and D-camphor $(2.5 \mathrm{~g} / 100 \mathrm{~g})$ as active ingredients. In traditional medicine, both fruits and leaves and flowers of different Crataegus species are used as cardioprotective agent and to support cardiovascular function (Shikov et al., 2014). However, according to the European Pharmacopoeia, Crataegi fructus may derive only from Crataegus laevigata and C. monogyna. In modern phytotherapy, Crataegus drugs are used to alleviate various cardiovascular conditions (e.g. arrhythmia, congestive heart failure). Interestingly, most commercial products with confirmed traditional use contain the leaves and flowers of the plants, and mono-preparations of Crategus species are used for lowering blood pressure (Assessment report on Crataegus spp., folium cum flore, 2016). Randomized, double-blind, placebo-controlled studies have confirmed its antihypertensive effects (Tassell et al., 2010). According to the European Medicines Agency's HMPC monograph, the use of Crataegus medicines containing 1-2 g of comminuted herbal substance per dose is based on their 'traditional use' to relieve symptoms of temporary nervous cardiac complaints (e.g. palpitations, perceived extra heart beat due to mild anxiety) (European Union herbal monograph on Crataegus spp., folium cum flore, 2016). Crataegus species are considered to be generally safe, but should not be recommended during pregnancy and lactation because of the lack of safety data. The topical use of camphor is known to increase local skin and muscle blood flow (Kotaka et al., 2014), but its effects on systemic blood pressure has not been studied in modern studies yet. The cardiotonic effect of camphor was observed in early studies (Saratikov et al., 1964). Interestingly, inhaled camphor is reported to decrease both heart rate and blood pressure (Eschlböck et al., 2017). Nevertheless, the combination of hawthorn and camphor has been used in the therapy to treat hypertension and the clinical efficacy has been assessed in human trials. The potential mechanism of action of this combination is unknown.
Although the effects of hawthorn and camphor combination on blood pressure and cognitive performance have been studied in randomized, controlled clinical trials (RCTs), their results are controversial and have not been reassessed statistically in a meta-analysis. Therefore, the aim of the present literature review and meta-analysis was to reassess and synthesize published evidence by systematically reviewing the available literature data on the efficacy of hawthorn-camphor combination based on randomized, controlled clinical studies. The following PICO (patients, intervention, comparison, outcome) format was applied: P: hypotensive and normotensive adult patients; I: hawthorn extract and camphor in combination; C: placebo; and O: changes in blood pressure and cognitive performance.

\section{Methods}

The meta-analysis was performed according to the PRISMA protocol, and it was registered in the International Prospective Register of Systematic Reviews (PROSPERO) on 29 May 2018 (registration number CRD42018095627).

\section{Information sources and search strategy}

Literature search was conducted until 24 September 2018, by using the following search strategy: [("crataegus"[MeSH Terms] OR "crataegus"[All Fields]) AND ("camphor"[MeSH Terms] OR "camphor"[All Fields])] for PubMed; [('hawthorn'/exp OR hawthorn) AND ('camphor'/exp OR camphor)] / [('crataegus'/exp OR crataegus) AND ('camphor'/exp OR camphor)] for Embase; [crataegus AND camphor / hawthorn AND camphor in Title, Abstract, Keywords in Trials] for Cochrane Central Register of Controlled Trials; and [TOPIC: (crataegus AND camphor / hawthorn AND camphor) Timespan: All years. Indexes: SCI-EXPANDED, SSCI, A\&HCI, ESCI.] for Web of Science. No language, publication date or publication status restrictions were applied. The reference lists of all identified articles were inspected. Only publicly available data were analysed, and neither the authors, nor the manufacturers were contacted for additional information.

\section{Eligibility criteria and study selection}

Randomized, placebo-controlled trials evaluating the effects of the combination of hawthorn extract and camphor in adult hypotensive and normotensive patients were included. Abstracts, case series, case reports and articles not reporting numerical data on efficacy were excluded. For reference management, Mendeley 1.17.9 was used. After removing duplicates, the remaining records were screened for eligibility based on the abstracts. The eligibility of the full texts of the resulting records was assessed by two reviewers (T.K., D.C.) independently. In case of disagreement between reviewers, a third reviewer (B.T.) was consulted.

\section{Data extraction and synthesis of the results}

Data collection was executed following the PRISMA guidelines. Study characteristics and results were extracted by the two reviewers independently. Discrepancies in extracted data were resolved by discussion. The following data items were extracted from the included papers: study design, characteristics of the patient population and sample size, intervention details, type of comparator(s), outcome measures and overall results. Systolic and diastolic blood pressure values and the results of a cognitive performance test (connect-the-numbers test) were extracted as outcome measures. Discrepancies in extracted data were resolved by discussion between the two reviewers.

\section{Risk of bias analysis}

The risk of bias was analysed by two of the authors (B.T., D.C.), 
using the Cochrane Collaboration tool which includes the following domains: random sequence generation, allocation concealment, blinding of participants and personnel, blinding of outcome assessment, incomplete outcome data, selective reporting and other scores of bias. For each domain, studies were judged to have a high (red), unclear (yellow) or low (green) risk of bias (see Supplementary Fig. S1). Disagreement was resolved by discussion. Risk of bias figures were prepared by using the RevMan 5 statistical program (Higgins JPT and Green S, 2011).

Quality of evidence

The Grading of Recommendations Assessment, Development and Evaluation (GRADE) was used for estimating the quality of evidence of all outcomes assessed (Guyatt et al., 2008).

\section{Statistical analysis}

The Hedges' $g$ statistic was applied to measure effect size for the difference between means, as it adjusts for bias in small samples by weighting the pooled standard deviations by sample size (Hedges, 1981). $p$-values of less than 0.05 were considered as indicators of significance. Data were graphically presented using forest plots.

Heterogeneity was assessed using the Cochran's Q test (Cochran, 1954) and Higgins' $I^{2}$ indicator (Higgins et al., 2003), where $\mathrm{Q}$ exceeds the upper tail critical value of Chi-square on $k-1$ degrees, and $I^{2}$ represents the percentage of effect size heterogeneity that cannot be explained by random chance. A p-value of less than 0.1 was considered suggestive of significant heterogeneity. According to the Cochrane Handbook (Higgins JPT and Green S, 2011), $I^{2}$ values of $25-50 \%, 50-75 \%$ and over $75 \%$ correspond to low, moderate and high degrees of heterogeneity, respectively. For statistical analysis we applied the fixed-effects model (Mantel-Haenszel method) (Mantel and Haenszel, 1959) on homogeneous results, whereas for heterogeneous results the random-effects model (DerSimonian-Laird method) (DerSimonian and Laird, 1986) was employed. All the statistical analyses were performed using the Comprehensive Meta-Analysis software (version 3, Biostat Inc., Englewood, NJ, USA).

\section{Results}

Study selection

Literature search was conducted in Embase, PubMed, the Cochrane Central Register of Controlled Trials and Web of Science databases, with Crataegus/hawthorn and camphor as search terms. After removing duplicates, 30 potentially relevant reports were collected (see excluded studies in Table 1S, Supporting Information). Eligible RCTs were selected according to the flow chart presented in Fig. 1.

After screening the abstracts, 8 publications were retrieved for fulltext screening (Belz et al., 2002; Belz and Loew, 2003; Erfurt et al., 2014; Hempel et al., 2005; Kroll et al., 2005; Schandry et al., 2018; Schandry and Duschek, 2008; Werner et al., 2009). Hempel et al. reported an epidemiological retrospective cohort study, not an RCT; therefore, it was not included in our meta-analysis (Hempel et al., 2005). Nevertheless, the authors of this study concluded that Korodin ${ }^{\circ}$ was safe and effective in the treatment of orthostatic hypotension. One review article (Belz and Loew, 2003) cited the results of a double-blind randomized clinical trial which was only reported as a conference abstract (Herrmann et al., 1996). According to this review (Belz and Loew, 2003) Herrmann et al. conducted a trial in which healthy male volunteers received sequential doses of camphor-hawthorn extract, and the administration of Korodin increased baseline mean arterial pressure (ANOVA $p<0.001$ ). However, this article was also excluded since it did not fulfil the inclusion criteria.

In total, seven studies, which were reported in six articles, were

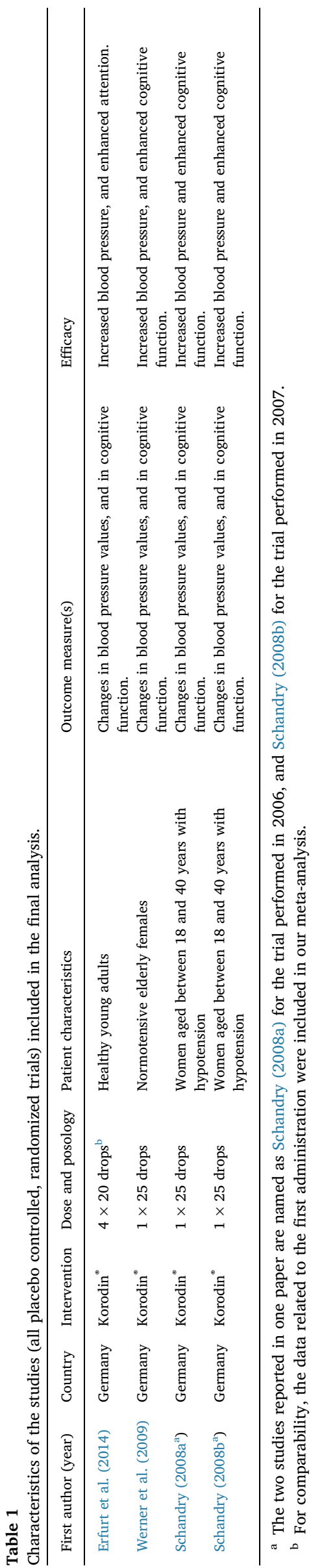




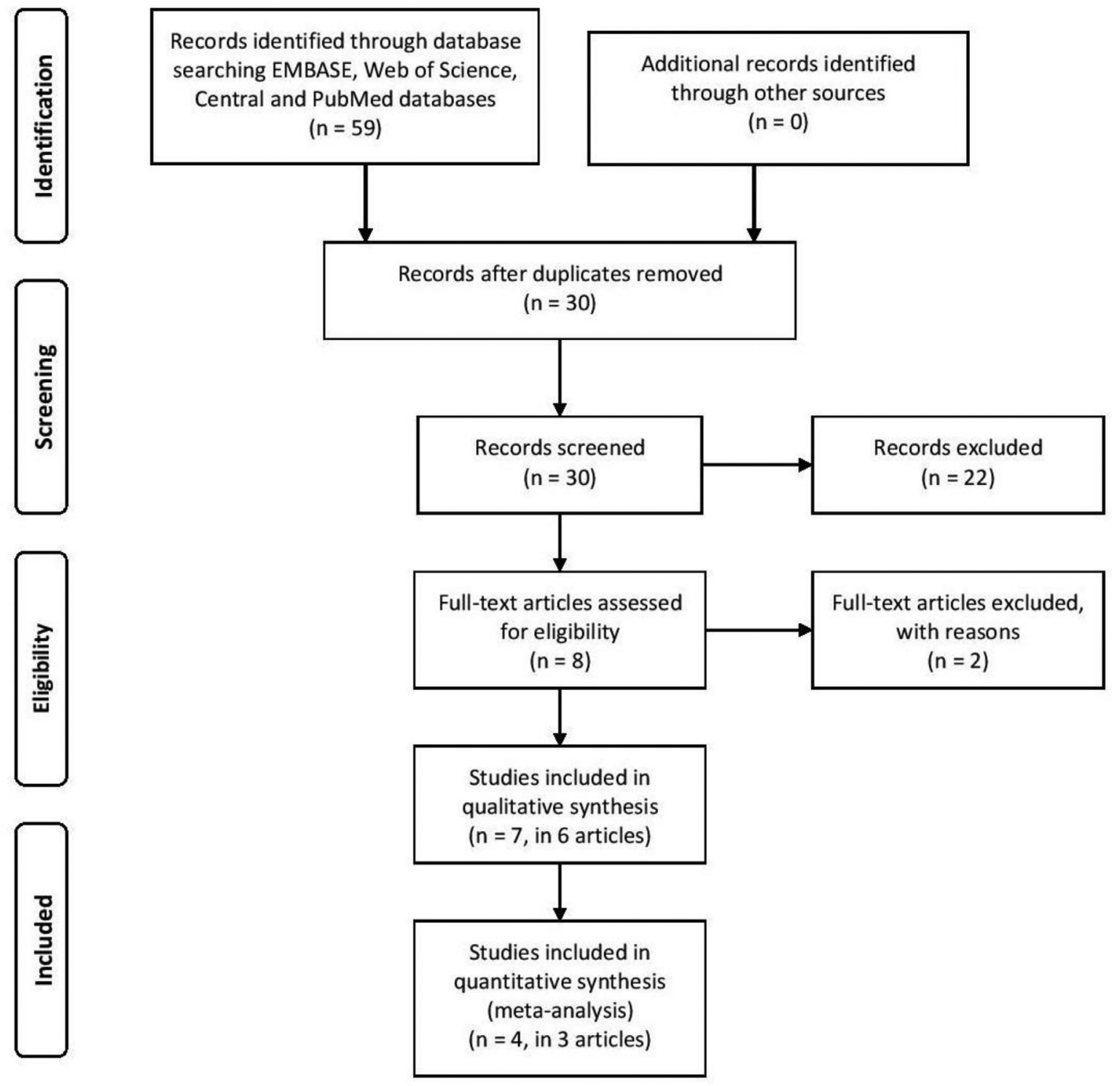

Fig. 1. PRISMA 2009 flow diagram for the identification of relevant studies.

selected for the qualitative analysis. Of these seven studies, three RCTs were finally not included in the statistical analysis. In a recently published paper, Schandry et al. studied the effects of Korodin ${ }^{\star}$ on adolescents (Schandry et al., 2018). Because the subjects in this study were not adults, it was not included in the quantitative analysis. In the RCT conducted by Belz et al. the clinical assessment substantially differed from that in other studies, hence it was not possible to compare the results of this study to the results of the other clinical trials (Belz et al., 2002). Another study failed to report all the essential data numerically, i.e. the means and standard deviations (SD) for systolic blood pressure (SBP), diastolic blood pressure (DBP), and mean arterial pressure (MAP) before and after the administration of placebo and Korodin were shown only graphically; therefore, the results of this trial could not be included in the quantitative analysis (Kroll et al., 2005). Finally, three articles, reporting 4 studies with 221 patients were assessed in the quantitative meta-analysis (Erfurt et al., 2014; Schandry and Duschek, 2008; Werner et al., 2009).

\section{Risk of bias assessment}

Overall, the methodical quality of the trials included in our final quantitative analysis was reckoned to be acceptable (Erfurt et al., 2014;
Schandry and Duschek, 2008; Werner et al., 2009), mostly with a low or unclear risk of bias (see Supplementary Figures S1 and S2). One paper reported on two trials (Schandry and Duschek, 2008), for which the methodology was similar but not exactly the same; therefore, the risk of bias analysis for these studies were performed separately. The first study was performed from 8 November 2006 to 31 November 2006, and it is marked as Schandry 2008a in Supplementary Figures S1 and S2; the second study, performed from 5 November 2007 to 5 December 2007, is marked as Schandry 2008b in Supplementary Figures S1 and S2.

Selection bias, i.e. random sequence generation and allocation concealment was considered to be high in the study reported by Erfurt et al., because the patients were randomized according to the sequence of their appearance (Erfurt et al., 2014). In both studies performed by Schandry and Duschek computer generated randomization plan was used; thus the selection bias of these studies was reckoned to be low (Schandry and Duschek, 2008). Werner et al. failed to describe the methods used for random sequence generation, allocation and blinding of outcome assessment; therefore the selection and detection bias of this study remains unclear (Werner et al., 2009).

Performance bias was unclear in all the included studies, because none of them described properly whether or not the intervention and 
the placebo were identical in shape, size, colour and odour. Detection bias differed between the studies. Erfurt and colleagues reported that unblinding occurred at data analysis; therefore this study has a high risk of detection bias. The other studies had an unclear risk of detection bias, because it was unclear from the papers whether outcome assessment was performed in a blinded manner or not.

All of the studies showed a low risk of attrition bias and an unclear risk of other types of bias. Reporting bias was reckoned to be high only in the study performed by Erfurt et al., because the results of the digit symbol test were not reported (Erfurt et al., 2014); nevertheless, the other studies were judged to have a low risk of reporting bias.

Due to the low number of studies, publication bias was not assessed by Egger's test, nor by funnel plots.

\section{Study characteristics}

All of the studies included in the meta-analysis were conducted by German authors and were presumably carried out in Germany (Erfurt et al., 2014; Schandry and Duschek, 2008; Werner et al., 2009). All were randomized, placebo-controlled, double blind studies. The study drug was Korodin ${ }^{\oplus}$ in all cases. All studies were supported by Robugen $\mathrm{GmbH}$ either by providing the test substances or by the financial remuneration of the participants. The papers were published between 2008 and 2014. The main characteristics of the included studies are summarized in Table 1 . The paper by Schandry and Duschek reports on two clinical trials (performed in 2006 and 2007, respectively). In both trials, women aged 18-40 years with hypotension SBP $<100 \mathrm{mmHg}$ were recruited, and the design of the studies were practically uniform. Exclusion criteria included pregnancy, hypothyroidism, severe physical illness, psychiatric disorders, psychoactive therapy, and taking cardiovascular medicine(s) or Korodin ${ }^{\circ}$. Each participant received a single dose of 25 drops of Korodin or placebo. The main difference between the two trials was that in the first one three cognitive tests (attentional performance test, connect-the-numbers test, alertness test), whereas in the second one two tests (connect-the-numbers test, digit symbol test) were performed. Blood pressure values (SBP and DBP) were measured prior to and after the application of Korodin ${ }^{\oplus}$ and placebo, similarly to performing the cognitive tests before and after the treatment. The time between Korodin ${ }^{\circ}$ application and blood pressure reading was $3 \mathrm{~min}$ in the first study, whereas it was not specified in the other one carried out in 2007 (Schandry and Duschek, 2008).

Werner and colleagues enrolled 80 normotensive female participants (50-80 years). Those taking medicines that affect the cardiac system were excluded. The applied dose of Korodin ${ }^{\circ}$ was 25 drops (single administration). SBP and DBP values were measured before and after the administration of the active treatment or placebo. In this study, the connect-the-numbers test and the digit symbol test were used to assess treatment effects on cognitive performance. Participants under psychopharmacological treatment $(n=3)$ were excluded from the cognitive tests, and regarding the connect-the-numbers test, those whose processing time was $>115 \mathrm{~s}$ were excluded from the analysis. MAP, continuous MAP and heart rate were also determined.

Erfurt and colleagues studied the effects of $4 \times 20$ drops of Korodin ${ }^{\oplus}$ in 54 healthy young adults (18-40 years, males and females). Exclusion criteria included affective disorders, serious chronic diseases, history of substance abuse, severe cognitive disorders, pregnancy and breastfeeding. The study drug or placebo was administered 4 times, with 10 min intervals. BP was measured 2 min after each administration. For comparability, only data obtained after the first administration were included in our meta-analysis. Cognitive performance was assessed using the $\mathrm{d} 2$ Test of Attention and the digit symbol test.

\section{Outcomes}

Excluded studies

Although three studies were excluded from the meta-analysis, the results of these may also contribute to the whole picture of the clinical efficacy of Korodin ${ }^{\circ}$. Belz et al. conducted two controlled, randomized, double-blind, crossover studies to assess the effects of three different single dosages ( 5 drops, 20 drops and 80 drops) of Korodin ${ }^{\circ}$ in a total of 48 young patients with orthostatic hypotension (average age: 25.6 and 27.3 years, respectively, in the two studies) (Belz et al., 2002). Their paper reports the results of these two separate trials, which were of the same design (except that phlebotomy was performed in the first study); however, the primary outcome measure was different in the two studies, being the change in SBP in the first one, and the change in MAP in the second one. Blood pressure (including SBP, DBP and MAP values) and HR were measured pre- and posttreatment, 1,3 and $5 \mathrm{~min}$ after the administration of Korodin ${ }^{\circ}$ or placebo, according to a tilt table test protocol. Accordingly, patients were lying on a table for $15 \mathrm{~min}$ before the table was lifted at an $80^{\circ}$ angle before $\mathrm{HR}$ and BP measurements to assess the effects of Korodin on orthostatic hypotension. Since no statistically significant efficacy of the treatment on the primary outcomes was detected in either studies, the authors pooled the data for a combined analysis. The combined analysis revealed a significant effect of the 80-drop-dose on increasing MAP 1 min after administration, as well as on increasing SBP after $1 \mathrm{~min}$ and DBP at all three posttreatment time points (1, 3 and $5 \mathrm{~min}$ ). There was a trend for correlation between dosage and efficacy, with the dose of 80 drops being the most effective (1 min post-administration, MAP, SBP and DBP increased by $4.48,6.21$ and $3.38 \mathrm{mmHg}$, respectively) (Belz et al., 2002).

Kroll et al. studied the effects of a single dose of 25 drops, and then 7 consecutive days of treatment with Korodin ${ }^{\circ}$ at a daily dose of $3 \times 25$ drops in 38 elderly ( $>50$ years) patients with orthostatic hypotension in a randomized, double-blind, placebo-controlled setting (Kroll et al., 2005). After a single application, the median decrease in MAP after standing up was $11.4 \mathrm{mmHg}$ for Korodin ${ }^{\circ}$ and $14.0 \mathrm{mmHg}$ for placebo. Compared to baseline, the median MAP improved by $4.3 \mathrm{mmHg}$ for Korodin $^{\circ}$ and $0.3 \mathrm{mmHg}$ for placebo. However, regarding the singledose application, these changes did not correspond to a statistically significant difference between the active and placebo arms. After 1 week of daily treatment the decrease in median MAP after standing up was $9.3 \mathrm{mmHg}$ for Korodin ${ }^{\circ}$ and $13.3 \mathrm{mmHg}$ for placebo (corresponding to an improvement of $5.9 \mathrm{mmHg}$ and $1.6 \mathrm{mmHg}$, respectively, compared to baseline), demonstrating a statistically significant difference in the efficacy of Korodin $v$ s. placebo. Regarding treatment effects on quality of life, assessed by an SF-12 questionnaire focusing on symptoms frequently associated with orthostatic hypotension, the active treatment was found to be superior in 5 over 7 items; however, the statistical analysis of these data was not published in the paper (Kroll et al., 2005).

A double-blind, randomized, placebo-controlled study by Schandry et al. aimed to assess the efficacy of a single dose of 20 drops of Korodin $^{\circ}$ on BP and cognitive performance (using the connect-thenumbers test and the digit symbol test) in 200 hypotensive adolescents (SBP $<118 \mathrm{mmHg}$ in boys and $<110 \mathrm{mmHg}$ in girls) (Schandry et al., 2018). SBP, DBP and MAP values increased significantly after the administration of 20 drops of Korodin ${ }^{\circ}$ compared to placebo $(p<0.001$, $p=0.004$ and $p<0.001$, respectively). Heart rate did not change significantly, and no difference in cognitive performance compared to baseline were detected based on the tests performed (Schandry et al., 2018).

\section{Included studies}

In the included studies the primary outcomes were changes in SBP and DBP from baseline (measured by the Riva-Rocci method and expressed in $\mathrm{mmHg}$ ) (Table 2). MAP was also calculated in two studies (Erfurt et al., 2014; Werner et al., 2009); however, this was not used as a measure of efficacy, since it is derived from SBP and DBP. Increases in these values directly reflect the efficacy of hypotension's therapy. Cognitive tests were used to assess the potentially favourable effects of Korodin ${ }^{\circ}$ on reduced cognitive performance which might be related to 
Table 2

Outcomes of the RCTs included in the meta-analysis.

\begin{tabular}{|c|c|c|c|c|c|c|c|c|c|}
\hline First author (year) & Group & Dose & Sample size & $\begin{array}{l}\text { Systolic blood } \\
\text { pressure before } \\
\text { treatment } \\
(\text { mean } \pm \mathrm{SD} \text {, } \\
\mathrm{mmHg})\end{array}$ & $\begin{array}{l}\text { Systolic blood } \\
\text { pressure after } \\
\text { treatment } \\
\text { (mean } \pm \mathrm{SD} \text {, } \\
\text { mmHg) }\end{array}$ & $\begin{array}{l}\text { Diastolic blood } \\
\text { pressure before } \\
\text { treatment } \\
(\mathrm{mean} \pm \mathrm{SD} \text {, } \\
\mathrm{mmHg})\end{array}$ & $\begin{array}{l}\text { Diastolic blood } \\
\text { pressure after } \\
\text { treatment } \\
(\text { mean } \pm \mathrm{SD} \text {, } \\
\mathrm{mmHg})\end{array}$ & $\begin{array}{l}\text { Connect the } \\
\text { numbers test } \\
\text { before } \\
\text { treatment (mean } \\
\pm \mathrm{SD}, \mathrm{s} \text { ) }\end{array}$ & $\begin{array}{l}\text { Connect the } \\
\text { numbers test } \\
\text { before treatment } \\
\text { (mean } \pm S D, s \text { ) }\end{array}$ \\
\hline \multirow[t]{2}{*}{ Erfurt et al. (2014) } & Korodin ${ }^{\circ}$ & 20 drops $^{b}$ & 38 & $109.8 \pm 12.1$ & $115.1 \pm 11.9$ & $73.7 \pm 8.5$ & $76.8 \pm 8.1$ & - & - \\
\hline & Placebo & & 15 & $115.2 \pm 14.9$ & $116.9 \pm 15.3$ & $72.1 \pm 6.9$ & $72.6 \pm 9.4$ & - & - \\
\hline \multirow[t]{2}{*}{ Werner et al. (2009 } & Korodin ${ }^{\circ}$ & 25 drops & 40 & $121.78 \pm 16.4$ & $127.23 \pm 17.21$ & $81.48 \pm 7.83$ & $86.53 \pm 8.94$ & $84.12 \pm 15.11$ & $71.93 \pm 13.58$ \\
\hline & Placebo & & 40 & $123.08 \pm 19.38$ & $125.7 \pm 19.23$ & $80.35 \pm 10$ & $81.68 \pm 10.14$ & $83.45 \pm 15.18$ & $74.6 \pm 14.82$ \\
\hline \multirow[t]{2}{*}{ Schandry $\left(2008 a^{a}\right)$} & Korodin ${ }^{\circ}$ & 25 drops & 19 & $97.65 \pm 5.11$ & $102.25 \pm 7.28$ & $62.2 \pm 6.89$ & $67.75 \pm 8.25$ & $67.7 \pm 12.8$ & $58.8 \pm 9.8$ \\
\hline & Placebo & & 21 & $96.6 \pm 4.83$ & $95.8 \pm 6.93$ & $60.84 \pm 5.53$ & $62.37 \pm 6.7$ & $60.4 \pm 9.4$ & $54.4 \pm 8.1$ \\
\hline \multirow[t]{2}{*}{ Schandry $\left(2008 b^{\mathrm{a}}\right)$} & Korodin ${ }^{\circ}$ & 25 drops & 24 & $99.08 \pm 5.59$ & $107.25 \pm 7.24$ & $64.46 \pm 6.58$ & $66.42 \pm 5.75$ & $63.8 \pm 14.5$ & $53.9 \pm 10.8$ \\
\hline & Placebo & & 24 & $101.67 \pm 7.38$ & $102.17 \pm 6.18$ & $63.79 \pm 6.62$ & $66.71 \pm 6.29$ & $59.1 \pm 11.1$ & $52.4 \pm 9.3$ \\
\hline
\end{tabular}

a The two studies reported in one paper are named as Schandry (2008a) for the trial performed in 2006, and Schandry (2008b) for the trial performed in 2007.

b For comparability, the data related to the first administration were included in our meta-analysis.

chronic hypotension. These tests focus on alertness, reaction time, processing speed, rule compliance, quality of performance, visualmotor coordination, and short-term visual memory. Three of the quantitatively analysed studies applied the connect-the-numbers test, hence these results were included in our meta-analysis. In the connectthe-numbers test, 90 numbers are arranged in an arbitrary sequence. Participants are asked to connect these numbers in the correct sequence as quickly as possible. The test comprises 4 sheets $(4 \times 90$ numbers $)$. Werner et al. defined exclusion criteria (processing time $>115 \mathrm{~s}$, psychopharmacological treatment) for the applicability of this test or being included in this specific analysis (Werner et al., 2009), whereas Erfurt et al. defined no such restrictions (Erfurt et al., 2014).

\section{Effects of Korodin on blood pressure}

In the study by Erfurt et al., 20 drops of Korodin ${ }^{\circledR}$ or placebo was administered to patients, 4 times in 10 min intervals. The active treatment was superior to placebo in terms of increasing SBP at the first 3 substance administrations, and in increasing DBP after the first administration (Erfurt et al., 2014). Werner et al. administered 25 drops of Korodin /placebo to their patients, and found that HR and MAP increased significantly at 2 and 3 min after administration of a single dose (Werner et al., 2009). In the two studies published by Schandry and Duschek, there was a significant increase in SBP in both studies, whereas DBP decreased significantly in the verum group (25 drops of Korodin ${ }^{\circ}$ ) compared to placebo only in study I (Schandry and Duschek, 2008). In summary, all the four included trials assessed the effects of Korodin on blood pressure, and the authors of each study concluded that the combined preparation increases blood pressure compared to placebo.

For the quantitative meta-analysis of data regarding the effects on DBP, a fixed-effects model $(Q=3.192$, df $=3, p=0.363$, $I^{2}=6.009 \%$ ) was applied: Hedges' $g=0.269$; lower limit of $95 \% \mathrm{CI}$ [LL]: 0.001, upper limit of 95\% CI [UL]: 0.538, $P$-value: 0.049 (Fig 2). For the statistical analysis of the results regarding the effects on SBP, a random-effects model was employed: $Q=7.691$, $\mathrm{df}=3, p=0.053$, $I^{2}=60.995 \%$. The results of our meta-analysis support that Korodin ${ }^{\circ}$ increases SBP: Hedges' $g=0.546$; lower limit of 95\% CI [LL]: 0.099, upper limit of 95\% CI [UL]: 0.992, p-value: 0.017 (Fig 3).

\section{Effects of Korodin on cognitive functions}

In the study by Schandry and Duschek, the efficacy of Korodin ${ }^{\circledR}$ was reflected in an improved cognitive performance of treated participants compared to placebo, in the connect-the-numbers test and the digit symbol test, but not in the alertness test and the attentional performance test (Schandry and Duschek, 2008). Erfurt et al. observed no effects attributable to Korodin ${ }^{\circ}$ in the digit symbol test; however, the d2 test of attention revealed significant efficacy at certain measurement points (Erfurt et al., 2014). Based on the findings of Werner et al., Korodin treatment significantly improved cognitive performance in the connect-the-numbers test and in the digit symbol test (Werner et al., 2009).

Three trials reported the results for the connect-the-numbers test; therefore, it was possible to perform a meta-analysis of these data. Applying a fixed-effects model $\left(Q=0.072, \mathrm{df}=2, p=0.965, I^{2}=0 \%\right)$ in our statistical analysis, we have confirmed that Korodin ${ }^{\circ}$ does not improve significantly the cognitive function of treated patients (Hedges' $g=0.276$; lower limit of $95 \%$ CI [LL]: -0.024 , upper limit of $95 \%$ CI [UL]: 0.576, p-value: 0.071 (Fig 4).

\section{Quality of evidence}

The grade of evidence of our statements was quantified with the GRADE approach (Table 3). Since only randomized controlled studies very included;the baseline grade of evidence was considered high. However, the authors think that bias-free high grade of evidence is only obtainable with analysing a large number of high quality randomized controlled studies. To assess the grade of evidence we considered five downgrading items (i.e. limitations in the design and implementation, indirectness, heterogeneity, imprecision, and publication bias).

\begin{tabular}{|c|c|c|c|c|c|c|c|}
\hline \multirow[t]{2}{*}{ Study name } & \multicolumn{7}{|c|}{$\underline{\text { Statistics for each study }}$} \\
\hline & $\begin{array}{l}\text { Hedges's } \\
\mathbf{g}\end{array}$ & $\begin{array}{l}\text { Standard } \\
\text { error }\end{array}$ & Variance & $\begin{array}{c}\text { Lower } \\
\text { limit }\end{array}$ & $\begin{array}{c}\text { Upper } \\
\text { limit }\end{array}$ & Z-Value & p-Valu \\
\hline Schandry, 2008 (b) & $-0,157$ & 0,284 & 0,081 & $-0,714$ & 0,401 & $-0,551$ & \\
\hline Erfurt, 2014 & 0,302 & 0,302 & 0,091 & $-0,289$ & 0,894 & 1,001 &, 3 \\
\hline Werner, 2009 & 0,385 & 0,224 & 0,050 & $-0,053$ & 0,824 & 1,724 & \\
\hline Schandry, 2008 (a) & 0,527 & 0,316 & 0,100 & $-0,092$ & 1,146 & 1,669 & 0,0 \\
\hline & 0,269 & 0,137 & 0,019 & 0,001 & 0,538 & 1,968 & \\
\hline
\end{tabular}

Hedges's g and $95 \% \mathrm{Cl}$

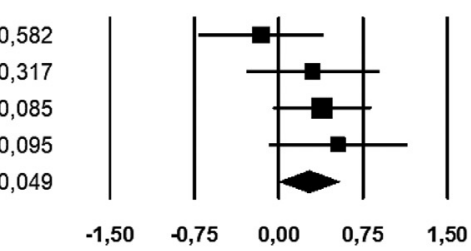

Fav. Placebo Fav. Verum

Fig. 2. The effects of the combined camphor-hawthorn extract on diastolic blood pressure in a fixed-effects model $(n=4)$. 


\begin{tabular}{lccrccccc} 
Study name & \multicolumn{7}{c}{ Statistics for each study } \\
\cline { 3 - 8 } & $\begin{array}{c}\text { Hedges's } \\
\text { g }\end{array}$ & $\begin{array}{c}\text { Standard } \\
\text { error }\end{array}$ & $\begin{array}{c}\text { Variance } \\
\text { Lower }\end{array}$ & $\begin{array}{l}\text { limit } \\
\text { limit }\end{array}$ & Z-Value & p-Value \\
Werner, 2009 & 0,154 & 0,222 & 0,049 & $-0,281$ & 0,588 & 0,693 & 0,489 \\
Erfurt, 2014 & 0,274 & 0,302 & 0,091 & $-0,317$ & 0,866 & 0,910 & 0,363 \\
Schandry, 2008 (a) & 0,746 & 0,321 & 0,103 & 0,116 & 1,375 & 2,321 & 0,020 \\
Schandry, 2008 (b) & 1,121 & 0,306 & 0,094 & 0,521 & 1,721 & 3,661 & 0,000 \\
& 0,546 & 0,228 & 0,052 & 0,099 & 0,992 & 2,395 & 0,017
\end{tabular}

Hedges's $\mathrm{g}$ and $95 \% \mathrm{Cl}$

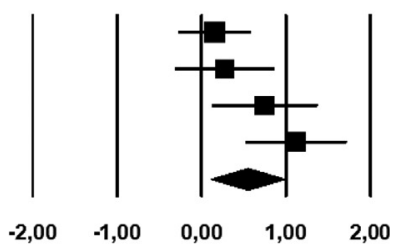

Fav. Placebo Fav. Verum

Fig. 3. The effects of the combined camphor-hawthorn extract on systolic blood pressure in a random-effects model $(n=4)$.

The finding that Korodin ${ }^{\circledR}$ increases SBP and DBP is supported by low quality evidence. Since selection, detection and reporting biases were considered to be high in the study of Erfurt et al., we have downgraded the level of evidence with one level (Erfurt et al., 2014). Moreover, publication bias might be suspected because the published evidence includes only a few small trials. Therefore, we have decided to downgrade the level of evidence with one more level. Overall, the blood pressure increasing effects of Korodin ${ }^{\circ}$ is supported by low quality of evidence, i.e. further research is very likely to have an important impact on our confidence in the estimate of effect and is likely to change the estimate.

It is supported by moderate quality of evidence that Korodin has a beneficial, but not statistically significant effect on the cognitive performance. We downgraded the high quality evidence due to high probability of publication bias, i.e. only three trials involving 168 patients studied the effects of Korodin ${ }^{\star}$ on the cognitive performance.

\section{Safety}

In the study by Belz et al., Korodin ${ }^{\circledR}$ was well tolerated and no adverse effects attributable to the treatment were reported. Clinical examinations and laboratory tests revealed no clinically relevant pathological findings; however, no detailed results were published (Belz et al., 2002). In the studies involving adolescents, no adverse events were reported (Schandry et al., 2018). In the study involving patients older than 50 years, only one adverse event was reported, which was not serious and was not related to the treatment (shoulder ache) (Kroll et al., 2005). Erfurt et al. did not report any adverse effects (Erfurt et al., 2014). Unfortunately, in two papers, no safety data were reported (Schandry and Duschek, 2008; Werner et al., 2009).

\section{Discussion}

Although hypotension is strongly associated with morbidity and mortality, its treatment options are much more limited than those of hypertension (Eschlböck et al., 2017). Besides a few synthetic drugs, there is only one combined natural product available as a medicine for the treatment of hypotension. The fixed combination of Crataegus extract and camphor (Korodin ${ }^{\circ}$ ) was introduced to the German market in
1927, and it is still used in Germany to relieve symptoms of orthostatic and chronic hypotension (Kroll et al., 2005). The present meta-analysis was designed to synthesize the currently available evidence for this product, and it is known to be the first systematic review and metaanalysis on this topic.

Based on a comprehensive literature search, four RCTs were identified, which included 221 adults. The effects of Korodin ${ }^{\circ}$ on the outcomes tested in RCTs are summarized in Table 2. Few publications reported on the same outcomes, and there were also differences in the posology of the study drug. Moreover, the setting of the trials was not uniform; therefore, the forest plots are short. Because of the scarce of trials reporting on the same outcomes it was not possible to properly assess publication bias by the Egger's tests or by funnel plots. Based on SBP and DBP results the combined preparation is confirmed to ameliorate hypotension, but its effects on cognitive performance is not significant. Therefore, based on our meta-analysis, the superiority of the combination of Crataegus extract and camphor over placebo in the treatment of hypotension is still not undoubtedly justified. These results are in line with the expectations reasoned by the low number of trials focusing on Korodin ${ }^{\oplus}$.

Limitations of our literature review and meta-analysis are largely related to the original studies. All the included trials were carried out in Germany, and there is a clear overlap between the authors of the papers. All of the quantitatively analysed trials were published within a relatively short time period, between 2008 and 2014. Because of the small number of participants, normotensive and hypotensive patients were analysed combined in our meta-analysis, yielding an inhomogeneous patient population, and it is possible that baseline blood pressure readings could have influenced the overall results.

Our meta-analysis, however, confirms the relative safety of Korodin ${ }^{\star}$, since the incidence of adverse events was not statistically different between the actively treated and the placebo groups. However, the number of patients involved in the reviewed trials are relatively low; hence a comprehensive evaluation of safety is required through further clinical studies and pharmacovigilance activities, which is indeed an unmet need, regarding that Korodin ${ }^{\circ}$ has been used for over 90 years.

\begin{tabular}{|c|c|c|c|c|c|c|c|}
\hline \multirow[t]{2}{*}{ Study name } & \multicolumn{7}{|c|}{ Statistics for each study } \\
\hline & $\begin{array}{l}\text { Hedges's } \\
\mathbf{g}\end{array}$ & $\begin{array}{l}\text { Standard } \\
\text { error }\end{array}$ & Variance & $\begin{array}{c}\text { Lower } \\
\text { limit }\end{array}$ & $\begin{array}{c}\text { Upper } \\
\text { limit }\end{array}$ & Z-Value & p-Value \\
\hline Werner, 2009 & 0,233 & 0,222 & 0,049 & $-0,203$ & 0,668 & 1,047 & 0,295 \\
\hline Schandry, 2008 (b) & 0,312 & 0,286 & 0,082 & $-0,248$ & 0,872 & 1,093 & 0,274 \\
\hline \multirow[t]{2}{*}{ Schandry, 2008 (a) } & 0,318 & 0,312 & 0,098 & $-0,294$ & 0,930 & 1,017 & 0,309 \\
\hline & 0,276 & 0,153 & 0,023 & $-0,024$ & 0,576 & 1,804 & 0,071 \\
\hline
\end{tabular}

Hedges's $\mathrm{g}$ and $95 \% \mathrm{Cl}$

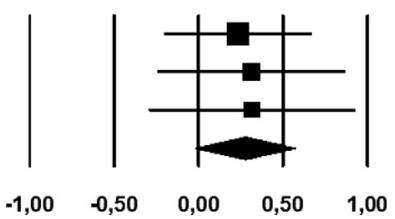

Fav. Placebo Fav. Verum

Fig. 4. The effects of the combined camphor-hawthorn extract on cognitive performance in a fixed-effects model $(n=3)$. 
Table 3

Summary of findings.

\begin{tabular}{|c|c|c|c|c|}
\hline Outcomes & No. of studies (patients) & Hedges' g ( $95 \%$ confidence interval; $\mathrm{p}$ value) & Quality of evidence & Comments \\
\hline SBP & 4 & $\begin{array}{l}\mathbf{0 . 5 4 6} \\
(0.099-0.992 ; 0.049)\end{array}$ & $\bullet \bullet \circ ०$ & Downgraded for risk of bias and publication bias \\
\hline DBP & $(221)$ & $\begin{array}{l}\mathbf{0 . 2 6 9} \\
(0.001-0.538 ; 0.017)\end{array}$ & low & \\
\hline Cognitive performance & $\begin{array}{l}3 \\
(168)\end{array}$ & $\begin{array}{l}\mathbf{0 . 2 7 6} \\
(0.024-0.576 ; 0.071)\end{array}$ & $\begin{array}{l}\text { ๑९९० } \\
\text { moderate }\end{array}$ & Downgraded for publication bias \\
\hline
\end{tabular}

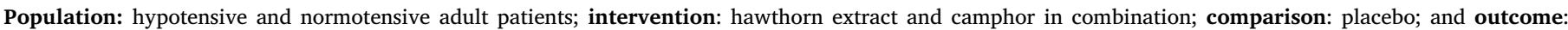
changes in blood pressure and cognitive performance.

\section{Conclusion}

Our meta-analysis supports that Korodin can be regarded as an effective complementary therapy for the treatment of hypotension; however, there is a need for further trials to improve the quality of the body of evidence. The optimum dose and duration of treatment is still unclear. In the included studies, Korodin ${ }^{\circ}$ was well-tolerated and its adverse effect profile did not differ from that of placebo. According to our meta-analysis, the combination of Crataegus extract and camphor increases SBP and DBP; however, its effects on cognitive performance is not significant. As a conclusion, there is a clear need for further highquality trials involving larger patient populations, and examining the effects of different, preferably higher doses of the combined preparation. Also it would be essential to apply comparable endpoints to assess the efficacy of Korodin ${ }^{\circ}$ with a lower risk of bias. In the future, further trials may allow the analysis of the effects of Korodin in dose-based subgroups. Our meta-analysis supports the long-standing use of Korodin; nevertheless, it highlights the lack of clinical data regarding natural products.

\section{Conflict of interest}

The authors declare no conflicts of interest.

\section{Acknowledgments}

The authors thank Dora Bokor, PharmD, for proofreading the manuscript. This study was supported by an Economic Development and Innovation Operative Programme Grant (GINOP 2.3.2-15-201600048 to P.H.) from the National Research, Development and Innovation Office, the János Bolyai Research Scholarship from the Hungarian Academy of Sciences (to D.C.), the project Development of Scientific Workshops of Medical, Health Sciences and Pharmaceutical Educations (EFOP-3.6.3-VEKOP-16-2017-00009), and a research grant (115796) from the National Research, Development and Innovation Office (to D.C.). T.L. was supported by the European Union and the State of Hungary, co-financed by the European Social Fund within the framework of the EFOP-3.6.1-16-2016-00008 project. L.M.C., S.T., G.V. and G.G. were supported by Hungarian Human Resources Development Operational Program (EFOP-3.6.2-16-2017-00006), and also by the Higher Education Excellence Program of the Hungarian Ministry of Human Capacities. The funding sources played no role in, nor influenced study design, data collection, data analysis, data interpretation, or preparation of the manuscript. The corresponding author had full access to all data processed in the study, and had the final responsibility for the decision to submit the study for publication.

\section{Supplementary materials}

Supplementary material associated with this article can be found, in the online version, at doi:10.1016/j.phymed.2019.152984.

\section{References}

Assessment report on Crataegus spp., folium cum flore(No. EMA/HMPC/159076/2014), 2016. . London.

Belz, G.G., Butzer, R., Gaus, W., Loew, D., 2002. Camphor-Crataegus berry extract combination dose-dependently reduces tilt induced fall in blood pressure in orthostatic hypotension. Phytomedicine 9, 581-588. https://doi.org/10.1078/ 094471102321616382.

Belz, G.G., Loew, D., 2003. Dose-response related efficacy in orthostatic hypotension of a fixed combination of D-camphor and an extract from fresh crataegus berries and the contribution of the single components. Phytomedicine 10 (Suppl 4), 61-67.

Cochran, W.G., 1954. The combination of estimates from different experiments. Biometrics 10, 101. https://doi.org/10.2307/3001666.

DerSimonian, R., Laird, N., 1986. Meta-analysis in clinical trials. Control. Clin. Trials 7, $177-188$.

Erfurt, L., Schandry, R., Rubenbauer, S., Braun, U., 2014. The effects of repeated administration of camphor-crataegus berry extract combination on blood pressure and on attentional performance - A randomized, placebo-controlled, double-blind study. Phytomedicine 21, 1349-1355. https://doi.org/10.1016/j.phymed.2014.06.014.

Eschlböck, S., Wenning, G., Fanciulli, A., 2017. Evidence-based treatment of neurogenic orthostatic hypotension and related symptoms. J. Neural Transm. 124, 1567-1605. https://doi.org/10.1007/s00702-017-1791-y.

European Union herbal monograph on Crataegus spp., folium cum flore(No. EMA/HMPC/ 159075/2014), 2016. . London.

Freeman, R., Wieling, W., Axelrod, F.B., Benditt, D.G., Benarroch, E., Biaggioni, I., Cheshire, W.P., Chelimsky, T., Cortelli, P., Gibbons, C.H., Goldstein, D.S.,

Hainsworth, R., Hilz, M.J., Jacob, G., Kaufmann, H., Jordan, J., Lipsitz, L.A., Levine, B.D., Low, P.A., Mathias, C., Raj, S.R., Robertson, D., Sandroni, P., Schatz, I., Schondorff, R., Stewart, J.M., van Dijk, J.G., 2011. Consensus statement on the definition of orthostatic hypotension, neurally mediated syncope and the postural tachycardia syndrome. Clin. Auton. Res. 21, 69-72. https://doi.org/10.1007/s10286011-0119-5.

Guyatt, G.H., Oxman, A.D., Vist, G.E., Kunz, R., Falck-Ytter, Y., Alonso-Coello, P., Schünemann, H.J., GRADE Working Group, 2008. GRADE: an emerging consensus on rating quality of evidence and strength of recommendations. BMJ 336, 924-926. https://doi.org/10.1136/bmj.39489.470347.AD.

Hedges, L.V., 1981. Distribution theory for glass's estimator of effect size and related estimators. J. Educ. Stat. 6, 107-128.

Hempel, B., Kroll, M., Schneider, B., 2005. [Efficacy and safety of a herbal drug containing hawthorn berries and D-camphor in hypotension and orthostatic circulatory disorders/results of a retrospective epidemiologic cohort study]. ArzneimittelForschung/Drug Res. 55, 443-450. https://doi.org/10.1055/s-0031-1296886.

Herrmann, V., Butzer, R., Roll, S., Malerczyk, C., Belz, G.G., 1996. Hemodynamic responses to a cumulative dosage of korodin herz-kreislauf-tropfen. Eur. J. Clin. Pharmacol. 50, 544.

Higgins, J.P.T., Thompson, S.G., Deeks, J.J., Altman, D.G., 2003. Measuring inconsistency in meta-analyses. BMJ 327, 557-560. https://doi.org/10.1136/bmj.327.7414.557.

Higgins, JPT, Green, S (Eds.), 2011. Cochrane Handbook for Systematic Reviews of Interventions Version 5.1.0 [updated March 2011]. The Cochrane Collaboration.

Jordan, J., Shannon, J.R., Biaggioni, I., Norman, R., Black, B.K., Robertson, D., 1998. Contrasting actions of pressor agents in severe autonomic failure. Am. J. Med. 105, $116-124$.

Kotaka, T., Kimura, S., Kashiwayanagi, M., Iwamoto, J., 2014. Camphor induces cold and warm sensations with increases in skin and muscle blood flow in human. Biol. Pharm. Bull. 37, 1913-1918.

Kroll, M., Ring, C., Gaus, W., Hempel, B., 2005. A randomized trial of Korodin ${ }^{\circ}$ herzkreislauf-tropfen as add-on treatment in older patients with orthostatic hypotension. Phytomedicine 12, 395-402. https://doi.org/10.1016/j.phymed.2004.11.003.

Mantel, N., Haenszel, W., 1959. Statistical aspects of the analysis of data from retrospective studies of disease. JNCI J. Natl. Cancer Inst. 22, 719-748. https://doi.org/ 10.1093/jnci/22.4.719.

Saratikov, A.S., Usov, L.A., Zhelnovich, L.I., 1964. [Role of the reinforcing nerve of the heart in the mechanism of the cardiotonic action of camphor]. Farmakol. Toksikol. 27, 439-444.

Schandry, R., Duschek, S., 2008. The effect of camphor-crataegus berry extract combination on blood pressure and mental functions in chronic hypotension - A randomized placebo controlled double blind design. Phytomedicine 15, 914-922. https:// doi.org/10.1016/j.phymed.2008.09.002.

Schandry, R., Lindauer, D., Mauz, M., 2018. Blood pressure and cognitive performance 
after a single administration of a camphor-crataegus combination in adolescents with low blood pressure. Planta Med. https://doi.org/10.1055/a-0634-6597.

Shikov, A.N., Pozharitskaya, O.N., Makarov, V.G., Wagner, H., Verpoorte, R., Heinrich, M., 2014. Medicinal plants of the russian Pharmacopoeia; their history and applications. J. Ethnopharmacol. 154, 481-536. https://doi.org/10.1016/j.jep.2014.04. 007.

Tassell, M.C., Kingston, R., Gilroy, D., Lehane, M., Furey, A., 2010. Hawthorn (Crataegus spp.) in the treatment of cardiovascular disease. Pharmacogn. Rev. 4, 32-41. https:// doi.org/10.4103/0973-7847.65324.

Victor, R.G., Talman, W.T., 2002. Comparative effects of clonidine and dihydroergotamine on venomotor tone and orthostatic tolerance in patients with severe hypoadrenergic orthostatic hypotension. Am. J. Med. 112, 361-368.

Werner, N.S., Duschek, S., Schandry, R., 2009. D-camphor-crataegus berry extract combination increases blood pressure and cognitive functioning in the elderly - A randomized, placebo controlled double blind study. Phytomedicine 16, 1077-1082. https://doi.org/10.1016/J.PHYMED.2009.05.006. 